\title{
Why Are Electron Capture Negative Ion Mass Spectra Not Reproducible? An Ion Source Problem
}

\author{
Voon S. Ong and Ronald A. Hites
}

School of Public and Environmental Affairs and Department of Chemistry, Indiana University, Bloomington, Indiana, USA

\begin{abstract}
Differences in the electron capture negative ion mass spectra of environmentally related organic compounds acquired on a VG 30-250 triple quadruple mass spectrometer and on an HP 5985B gas chromatography/mass spectrometry system were investigated with respect to the ion formation process. Neither ion source temperature nor pressure was responsible for the differences. The populations of thermal electrons in both ion sources were experimentally determined and found to be similar, suggesting that electron capturing reactions should proceed with comparable efficiencies in both ion sources. The ion extraction efficiencies of the two instruments were examined by monitoring the transmission profiles of low- and high-mass ions as a function of lens potentials. Results indicated that the HP 5985B extraction lens significantly suppressed low-mass ions. Further, theoretical evaluation of ion trajectories using SIMION suggested that on the HP 5985B, low-mass ions entered the mass analyzer as a defocused beam, but high-mass ions entered the analyzer as a well-collimated beam. On the VG 30-250, low- and high-mass ions were transmitted to the analyzer with equal efficiency by the ion extraction system. (J Am Soc Mass Spectrom 1993, 4, 270-277)
\end{abstract}

$\mathrm{E}$ lectron capture negative ionization (ECNI) mass spectrometry is a particularly sensitive and selective tool for the trace determination of electrophilic organic environmental contaminants [1-11]. This technique, however, has not enjoyed widespread popularity because it lacks reproducibility among different instruments [12-14]. Some researchers have reported spectra dominated by low-mass ions, whereas others have observed spectra showing intense molecular anions for the same compounds [14]. This problem is further complicated by nonstandardized temperature and pressure monitoring devices on different commercial instruments. Accurate measurement of these parameters is important because optimum ECNI sensitivities often require careful control of both the ion source temperature and the pressure of the enhancement gas. Mass spectral differences can also be caused by variations in ion formation and extraction processes, in ion transmission to and through the mass analyzer, and in ion detection as a function of mass [15]. Of these factors, ion formation and extraction processes are the least understood, particularly among the different ion source configurations and extraction optics used by commercial instruments.

Address reprint requests to Ronald A. Hites, School of Public and Environmental Affairs and Department of Chemistry, Indiana University, Bloomington, IN 47401.
In the present study, the performance of a VG 30-250 triple quadrupole mass spectrometer (only the first analyzer was used) operating under ECNI conditions was compared with that of a Hewlett-Packard (HP) 5985B gas chromatography/mass spectrometry (GC/MS) system. The HP instrument has proved to be reliable for ECNI work over the course of many years; however, results from a previous ECNI mass spectral reproducibility study suggested that the $\mathrm{HP}$ instrument produced unusually low signals for low-mass ions, such as $\mathrm{Cl}^{-}$[14]. No clear explanation of this observation was advanced, and we resolved to provide one.

\section{Experimental}

\section{Chemicals}

Environmentally related (halogenated and nonhalogenated) organic compounds were ohtained from various sources (EPA Health Effects Research Laboratory, Office of Research and Development, Research Triangle Park, NC; Aldrich Chemical Co., Milwaukee, WI; Chem Service, West Chester, PA; Analabs, Inc., North Haven, CT). Standards with concentrations ranging $10-100 \mu \mathrm{g} / \mathrm{mL}$ were prepared in hexane. Serial dilutions were carried out to obtain the appro- 
priate concentration for GC injection; typically, 0.1$2.0 \mathrm{ng}$ was injected in $1 \mu \mathrm{L}$ of solution.

\section{Instrumentation}

ECNI mass spectra were recorded on a VG $30-250$ triple quadrupole mass spectrometer ( $V G$ Instruments Inc., Danvers, MA) equipped with an HP $5890 \mathrm{GC}$. Samples were introduced by a $30 \mathrm{~m} \times 0.25 \mathrm{~mm}$ DB-5 fused silica column (J \& W Scientific, Rancho Cordova, CA) that was inserted directly into the ionization chamber. Injections were made on column. Various column temperature programs were used; a typical program was $30-250{ }^{\circ} \mathrm{C}$ at $30^{\circ} \mathrm{C} / \mathrm{min}$ with a $10-\mathrm{min}$ hold at $250^{\circ} \mathrm{C}$. Helium was used as the carrier gas (at $40 \mathrm{~cm} / \mathrm{s}$ ), and the transfer line temperature was $280^{\circ} \mathrm{C}$ at all times. Methane $(99.99 \%$ purity; Air Products, Allentown, PA), which was used as the enhancement or reagent gas, flowed into the ionization chamber from a separate gas line at a rate sufficient to achieve the desired ion source pressure.

The ion source block used for ECNI work was a modified VG electron-impact ion source block. The rectangular-shaped volume was replaced with a smaller, cylindrically shaped ECNI ionization chamber, measuring $3 \mathrm{~mm}$ in height by $10 \mathrm{~mm}$ in diameter. (A theoretical study carried out by Siegel [161 predicted that best sensitivity for ECNI work is obtained with a small ion source volume.) Because only the volume of the ionization chamber was changed, the ion extraction optics were kept the same for negative ion work. Ions exit the chamber through a flat plate with an exit aperture of $1-\mathrm{mm}$ diameter. The end opposite to the ion exit plate in the cylindrical ionization chamber was fitted with a repeller. The pressure inside the innization chamber was measured directly with a Pirani gauge (calibrated with methane), which was plugged into a hole on the side of the ion source block. Optimum sensitivity was obtained at a pressure of 0.30 torr. The ion source temperature was monitored by a thermocouple in direct contact with the source block; mass spectra were normally obtained at a source temperature of $100^{\circ} \mathrm{C}$.

Instrument tuning and mass axis calibration were carricd out using a mixture $(1000: 1)$ of perfluorotributylamine (PFTBA; PCR Research Chemicals, Inc., Gainesville, FL) and pentafluorobenzonitrile (PFBN; PCR Research Chemicals, Inc.). The mixturc was introduced neat into the ionization chamber through a heated $\left(170^{\circ} \mathrm{C}\right)$ septum inlet that provided a constant bleed of the tuning mixture at a depletion rate of approximately $1 \% / \mathrm{h}$. The instrument was tuned with the $\mathbf{M}^{-}$ion $(m / z 193 ; 5-15 \%$ of $m / z$ 633) of PFBN and the $\left[\mathrm{M}-\mathrm{C}_{4} \mathrm{~F}_{9}\right]^{-}$ion $(\mathrm{m} / z$ 452; 20-40\% of $\mathrm{m} / z$ 633) and the $\left[\mathbf{M}-\mathbf{F}_{2}\right]^{-}$ion $(m / z 633 ; 100 \%$ relative abundance) of PFTBA. Calibration of the mass axis used the $m / z 193$ ion of PFBN and the $m / z$ 414, 452, 595 , and 633 ions of PFTBA. The enhancement gas was ionized with a $120-\mathrm{eV}$ beam of primary electrons with an electron emission current of $300 \mu \mathrm{A}$.
An HP 5985B GC/MS system (Hewlett Packard, Palo Alto, CA) was also used in this study. This is a quadrupole mass spectrometer equipped with a combined electron impact and chemical ionization source [17]. The jet separator had been replaced with a modified transfer line to allow the capillary GC column to be inserted directly into the ion source; a direct inlet internal standard line had been added; and the opening where the direct insertion probe entered the source had been fitted with a capacitance manometer (MKS Instruments, Burlington, MA). Methane flowed coaxially around the capillary column and through a transfer line heated to $280^{\circ} \mathrm{C}$ before entering the ion source. Samples were introduced using the same type of column and carrier gas (operating at the same velocity) as the VG instrument. Injections were made in the splitless mode using a similar temperature program as the VG 30-250. Ion source pressure measurements were made with the capacitance manometer; source temperatures were monitored by a thermocouple in direct contact with the ion source block. The typical operating pressure and temperature were 0.40 torr and 100 ${ }^{\circ} \mathrm{C}$, respectively. The instrument was tuned (using the same tuning criteria) and calibrated using 1000:1 PFTBA/PFBN. The emission current was typically held at $300 \mu \mathrm{A}$, and the electron energy was $200 \mathrm{eV}$.

\section{Results and Discussion}

The mass spectra of chlorinated compounds acquired on the VG 30-250 are dominated by an abundant $\mathrm{Cl}^{-}$ ion, whereas the spertra acquired on the HT 598.5B show very much less $\mathrm{Cl}^{-}$. An example of this disparity was observed in the mass spectra of endosulfan (molecular weight 404) acquired on the two instruments. In the spectrum recorded on the VG 30-250 (Figure 1, top), an intense $\mathrm{Cl}^{-}$peak is present, whereas a much less intense $\mathrm{Cl}^{-}$peak is seen in the $\mathrm{HP}$ 5985B mass spectrum (Figure 1, bottom). The molecular ion is observed in both spectra at $m / z-404$, and other fragment ions are seen at $m / z-370$ owing to [M $\mathrm{Cl}+\mathrm{H}^{-}$and at $m / z-235$ owing to $\mathrm{C}_{5} \mathrm{Cl}_{5}^{-}$. Figure 1 represents the mass spectra of endosulfan obtained on the two instruments under typical operating conditions. We can "adjust" the mass spectral appearance on a given instrument by operating under atypical and less than optimum conditions; however, we could never force the spectra from the two instruments to become similar. For example, it was possible to increase the ratio of $\mathrm{Cl}^{-}$to $\mathrm{M}^{-}$for the $\mathrm{HP} 5985 \mathrm{~B}$ spectrum to almost $1: 1$ by decreasing the ion source pressure (at the expense of losing sensitivity by almost 60 times). The typical ratio of $\mathrm{Cl}^{-}$to $\mathrm{M}^{-}$for the VG 30-250 spectrum was $5: 1$, and it was possible to decrease this ratio to $3: 1$ by increasing the source pressure on the VG instrument (again losing sensitivity); however, even this ratio is still not near that of the $\mathrm{HP}^{\mathrm{P}}$ instrument.

Our observation of different relative abundances of low- to high-mass ions in the ECNI spectra acquired 

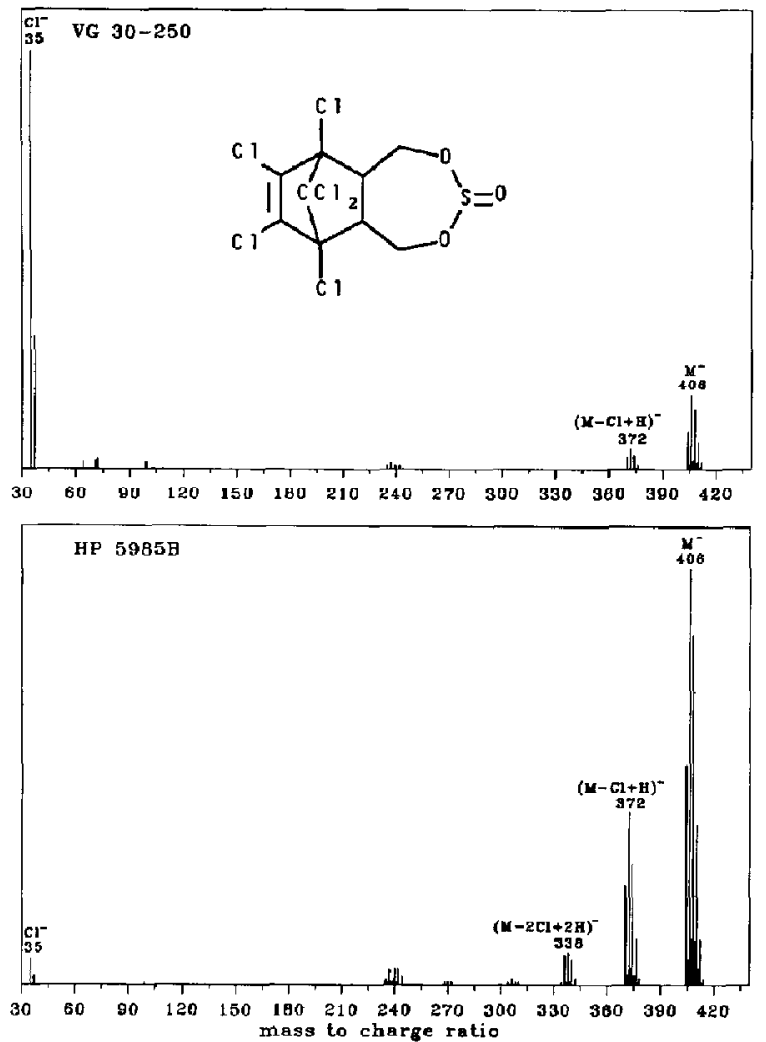

Figure 1. Mass spectra of endosulfan (200 pg injected) recorded on the VG 30-250 (top) and HP 5985B (bottom) instruments.

on different instruments was not unique. Previously, our laboratory had partiripated in an interlaboratory study to compare the mass spectra of a number of compounds recorded on different commercial instruments under a predetermined set of operating conditions [14]. Among the instrument types were the HP 5985B (current system), Finnigan 4500 (Finnigan-MAT, San Jose, $(A)$, and two magnetic sector instruments (an MS-80 and a VG 70-70). A comparison of the mass spectra of endosulfan and decafluorotriphenylphosphine (DFTPP) from these instruments showed that although the specific ions detected were the same on the different instruments, relative ion abundances varied dramatically. The dissimilarities were most distinct between spectra from the HP $5985 \mathrm{~B}$ and the rest of the instruments used in the study. The abundances of the $\mathrm{Cl}^{-}$from endosulfan and of $m / z-167\left(\mathrm{C}_{6} \mathrm{~F}_{5}^{-}\right)$ from DFTPP were significantly lower in the spectra acquired on the HP instrument compared with those obtained from all other mass spectometers.

On the basis of the mass spectra from this interlaboratory study and on spectra acquired on the VG instrument, we suspected that the VG ion source was operating in a manner similar to the other instruments but that the HP 5985B was operating "unconventionally." In other words, the ion source on the HP instrument produced primarily molecular ions, in contrast to other instruments, which showed predominantly low-mass ions (such as $\mathrm{CH}^{-}$). What could cause such a difference? There are two explanations: (1) More nondissociative resonance capture is occurring (thereby producing more molecular ions and high-mass ions) in the ion source of the HP instrument as a result of a lower electron energy distribution; and (2) similar amounts of low- and high-mass ions were actually produced in the ion sources, but the differences in the recorded mass spectra were due to suppression of low-mass ions by the HP instrument. If the former was occurring, then an understanding of the processes that control the production of a lower electron energy distribution could enhance the reproducibility of ECNI spectra among different instrument types.

\section{Electron Energy Distribution}

Negative ion mass spectral variations can be caused by differences in electron energy distributions because maximum cross sections for electron capture reactions occur in a narrow energy range, particularly for nondissociative electron attachment processes [18]. Differences in electron energy distributions can be caused by variations in ion source temperature, pressure, and the presence of electric fields. It was difficult to estimate the electric fields that could be present in the source region and their effects on electron energies. Therefore, we chose to directly examine the electron energy distribution in each ion source. Although a complete measure of the electron energy distribution in the ion source was beyond the capabilities of these commercial instruments, qualitative evidence can be obtained by using a procedure outlined by Hunt and Crow [19].

Briefly, operation of a mass spectrometer under methane chemical ionization conditions produces a mixture of positive reagent ions and a population of secondary electrons with near-thermal energies (eq 1). The positive reagent ions, $\mathrm{CH}_{5}^{+}$and $\mathrm{C}_{2} \mathrm{H}_{5}^{+}$, are mainly produced as indicated by eqs 2 and 3 . The electron energy distribution in both the VG and $\mathrm{HP}$ instrument ion sources was examined under typical operating conditions by comparing the maximum signal generated by the positive methane reagent ions, $\mathrm{CH}_{5}^{+}$and $\mathrm{C}_{2} \mathrm{H}_{5}^{+}$, with that produced by negatively charged sample molecules.

$$
\begin{aligned}
2 \mathrm{CH}_{4}+2 \mathrm{e}^{-} \rightarrow & \mathrm{CH}_{4}^{+}+\mathrm{CH}_{3}^{+}+\mathrm{H} \\
& +2 \mathrm{e}^{-} \text {(secondary) }+2 \mathrm{e}^{-} \text {(primary) } \\
\mathrm{CH}_{4}^{+}+\mathrm{CH}_{4} \rightarrow \mathrm{CH}_{5}^{+}+\mathrm{CH}_{3} & \mathrm{CH}_{3}^{+}+\mathrm{CH}_{4} \rightarrow \mathrm{C}_{2} \mathrm{H}_{5}^{+}+\mathrm{H}_{2}
\end{aligned}
$$

Sulfur hexafluoride $\left(\mathrm{SF}_{6}\right)$ has been used as an effective electron scavenger $[20,21]$. Interaction of $\mathrm{SF}_{b}$ with low-energy electrons mainly produces two ions, $\mathrm{SF}_{6}^{-}$ and $\mathrm{SF}_{5}^{-}$. Formation of $\mathrm{SF}_{6}^{-}$occurs at a narrow electron energy range, $0.0 \pm 0.03 \mathrm{eV}$, whereas formation of $\mathbf{S F}_{5}^{-}$ 
peaks at $0.15 \mathrm{eV}$ and falls to nearly zero at energies above $1 \mathrm{eV}$. Using the procedure outlined by Hunt and Crow [19], an estimate of the electron energy distribution in each ion source was obtained from the ratios of $\mathrm{SF}_{6}^{-}$and $\mathrm{SF}_{5}^{-}$to the total positive reagent gas ions.

The results obtained on the VG instrument indicated that approximately $55 \%$ of the electrons in its ion source have energies close to $0.0 \mathrm{eV}$, and another $6 \%$ have energies of approximately $0.2 \mathrm{eV}$. Comparable numbers were obtained for the HP 5985B: 58\% have energies near $0.0 \mathrm{eV}$, and $6 \%$ have energies of approximately $0.2 \mathrm{eV}$. This suggested that for both ion sources operating under typical conditions, electron capturing reactions should proceed with similar efficiencies.

\section{Comparison of Detection Limits}

During the course of this study, it was noticed that if both mass spectrometers were set to scan a mass range from 200 to $430 \mathrm{Da}$, the resulting spectra of endosulfan were nearly identical. On the basis of this observation, we hypothesized that similar abundances of high-mass ions were actually produced in each ion source, and as a result, the limits of detection would be the same on both instruments when only high-mass ions were considered. Clearly, the comparison of detection limits is only an approximate method because other factors (sample introduction method, ion extraction and transmission efficiency, detector response, and background noise) may also influence the outcome. Nevertheless, experimental results indicated that both mass spectrometers had comparable limits of detection for endosulfan (5 pg full scan at a signal-to-noise ratio of 5) when only the high-mass ions ( $>200 \mathrm{Da}$ ) were used for quantitation. This result indicated that similar amounts of high-mass ions are formed in both ion sources.

\section{Ion Source Temperature and Pressure}

Instrumental parameters that may influence relative ion abundances in an ECNI mass spectrum include ion source temperature and pressure, sample concentration, and type of erhancement gas [15]. Because the amount of sample introduced was approximately the same and because methane was used as the enhancement gas in both instruments, these two factors were excluded from consideration. The following experiments on ion source temperature and pressure were carried out to determine whether atypical instrumental conditions could produce similarity in mass spectra acquired on the VG 30-250 and HP 5985B.

Past studies have shown that ion source temperature strongly influences the appearance of an ECNI mass spectrum [22-24]. In general, it has been found that higher ion source temperatures increased the abundance of fragment ions such as $\mathrm{Cl}^{-}$, whereas lower temperatures enhanced molecular ion abundance. Because ECNI operation often requires careful control of ion source temperature, differences in the manner by which ion source temperature measurements are made by various commercial instruments have been blamed for mass spectral variations [14]. Because both the VG and HP instruments monitor source temperatures using thermocouples located directly on the respective ion source blocks, source temperature measurement error probably could not have caused mass spectral variations between the two instruments. Nevertheless, we conducted a study to determine the overall effects of varying ion source temperatures. The VG ion source temperature was lowered to $50{ }^{\circ} \mathrm{C}$ to see whether a low ion source temperature would suppress the generation of lowmass ions, particularly $\mathrm{Cl}^{-}$, and enhance high-mass ions.

Two compounds were chosen for this VG ion source temperature experiment-endosulfan and DFTPP. Endosulfan represented a chlorinated compound for studying the effects of ion source temperature on the relative abundances of low-mass ions $\left(\mathrm{Cl}^{-}\right)$. Unlike endosulfan, the DFTPP spectrum on both instruments consisted of a dominant $\mathrm{M}^{-}$at $m / z-442$. Therefore, DFTPP is excellent for studying the effects of ion source temperature on a stable molecular ion. Variations in the relative ion abundances for the two compounds were measured as a function of ion source temperature. The plot for endosulfan (Figure 2, top) indicated that the relative abundance of the molecular ion $(m / z-406)$ was approximately $25 \%$ at a VG ion source temperature of $50^{\circ} \mathrm{C}$, but even at a source temperature as low as $50^{\circ} \mathrm{C}, \mathrm{Cl}^{-}$was still the most intense peak in the mass spectrum. The data for DFTPP (Figure 2, bottom) suggested that the molecular ion remained the most intense peak in the mass spectrum unless the temperature exceeded $160{ }^{\circ} \mathrm{C}$. Relative abundances of different ions did not vary significantly between 50 and $100^{\circ} \mathrm{C}$. From this ion source temperature study, we found that low-mass endosulfan ions predominated even at low ion source temperatures on the VG instrument. Even the increase in the molecular ion abundance relative to $\mathrm{Cl}^{-}$shown by endosulfan at $50{ }^{\circ} \mathrm{C}$ cannot account for the disparity between lowand high-mass ion abundances observed from the two instruments.

Ion source pressure has been found to affect relative ion abundances to a certain extent $[15,25,26]$. Increasing the source pressure may increase the degree of electron thermalization and may lower mean electron energies, thus producing more electron allachment. Increasing the pressure also increases the stabilization of excited molecular ions through collisions, which would ultimately result in extending the lifetimes of the negative ions with respect to autodetachment. Collisional stabilization of the molecular ions may also suppress other electron-molecule reactions, such as dissociative electron capture.

A study of the relative abundance of various ions of PFTBA as a function of ion source pressure on the VG 

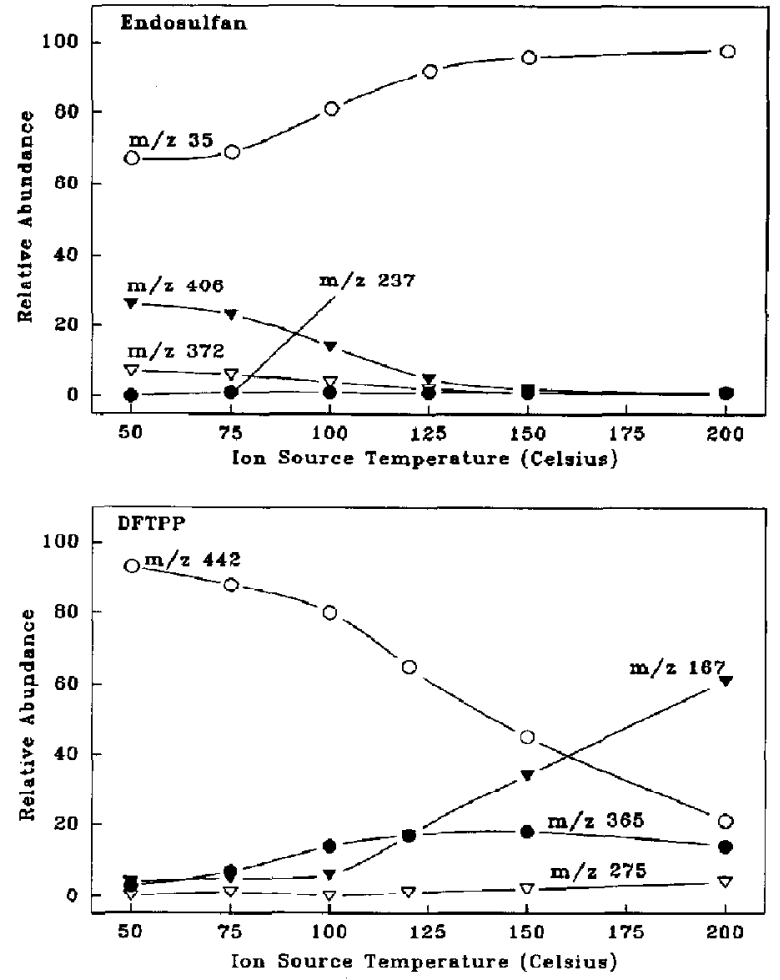

Figure 2. Relative abundances of different ions of endosulfan (top) and DFTPI' (battom) as a function of ion source temperature of the VG 30-250 instrument.

instrument indicated that there was no change in the relative abundances of the ions above a pressure of 0.20 torr. For DFTPP, mass spectra recorded on the VG instrument at pressures between 0.30 and 0.80 torr showed negligible differences. The ratio of $\mathrm{Cl}^{-}$to $\mathrm{M}^{-}$ of endosulfan decreased to 3:1 from its typical value of 5:1 when the $V G$ ion source pressure was raised from 0.30 to 0.80 torr, although sensitivity is optimum at 0.30 torr; however, even a large increase $(>100 \%)$ in the VG ion source pressure could not produce an enhancement in the molecular ion sufficient to account for the differences between the mass spectra from the VG and HP instruments.

These results showed that ion source pressure has pronounced effects on sensitivity [26]. Below a certain critical pressure, there is probably inefficient electron thermalization, resulting in decreased sensitivity. Total ion current versus ion source pressure plots for all three test compounds indicated that sensitivity was at a maximum at a pressure of approximately 0.30 torr on the VG instrument. The initial increase in ion current (from 0.10 to 0.30 torr) is probably due to increased electron thermalization and collisional stabilization, whereas at higher pressures, the decline in the ion current is probably due to ion beam scattering $[26,27]$. Consequently, the ion source pressure was carefully monitored and maintained at 0.30 torr for all ECNI experiments on the VG instrument.

In a separate experiment, the ion source pressure of the HP 5985B was varied throughout the range accessible to observe its effects on mass spectral appearance. It was found that above a pressure of 0.21 torr, there was no significant difference in the relative ion abundances. For example, the ratio of $\mathrm{Cl}^{-}$to $\mathrm{M}^{-}$for endosulfan was typically approximately $1: 10$. Although relative abundances remained similar, there was a drastic decrease in sensitivity when the mass spectrometer was operated at an ion source pressure of 0.21 torr (by approximately seven times). Sensitivity was typically optimum at 0.40 torr. Consequently, this pressure was always carefully maintained on the HP instrument. At a low ion source pressure of 0.18 torr (the source pressure was 0.13 torr without any enhancement gas), the ratio of $\mathrm{Cl}^{-}$to $\mathrm{M}^{-}$abundances reached 1:1 (at the expense of decreased sensitivity). This ratio, however, still failed to account for the differences between the mass spectra from the VG and $\mathrm{HP}$ instruments (typical ratio of $\mathrm{Cl}^{-}$to $\mathrm{M}^{-}$abundances of endosulfar on the VG instrument mass spectrum was $5: 1$ ).

\section{Collimating Magnetic Field Strength}

The HP 5985B uses an unusually high magnetic field for electron beam collimation $(360 \mathrm{G}$, as measured in the region of the ionization chamber) [28]. It is possible that low-mass ions such as $\mathrm{Cl}^{-}$could be affected by these magnetic fields, and this could cause the differences observed in the relative abundance of low- and high-mass ions. An experiment was developed to test this idea. The normal magnets used on the HP instrument were replaced with a weaker set of magnets (190 G) acquired from the manufacturer [28]. The weaker magnetic field strength was comparable to that used on the VG instrument $(200 \mathrm{G})$. Mass spectra of endosulfan acquired on the $H P$ instrument fitted with the weaker magnets were almost exactly the same as mass spectra recorded with the normal magnets. In both cases, a very low abundance of $\mathrm{Cl}^{-}$was detected. This experiment was also carried out in the extreme case: The collimating magnets were removed from the instrument altogether. Although sensitivity suffered owing to poor primary electron collimation, the results were the same: There was no difference in the mass spectrum of endosulfan; $\mathrm{Cl}^{-}$was still of very low abundance.

\section{Ion Extraction Optics}

Very little is known about the effect of the optics used in the VG 30-250 and HP 5985B instruments to control the extraction and transmission of ions to the mass analyzers. We had assumed that the respective ion extraction optics had equivalent transmission efficiencies. An electric field is normally applied between 

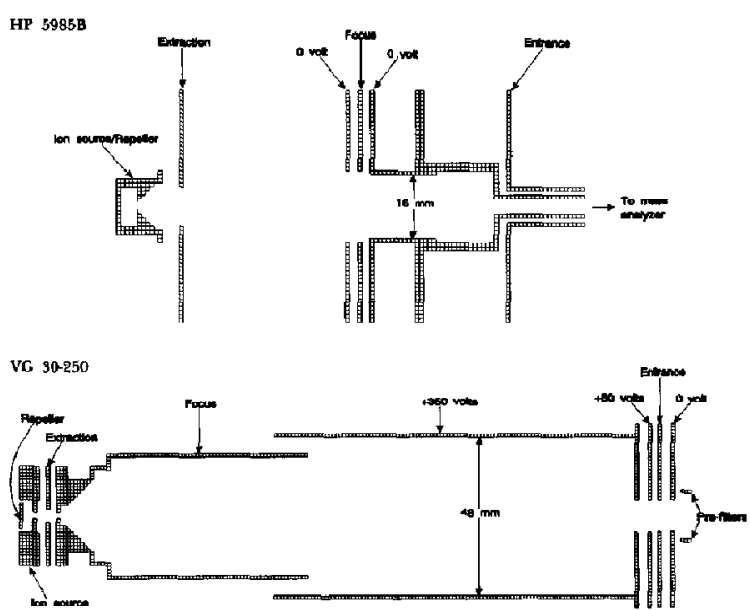

Figure 3. Ion source and associated ion extraction optics of the HP 5985B (top) and the VG 30-250 (bottom) instruments. Scale: each grid unit is $1 \mathrm{~mm} \times 1 \mathrm{~mm}$.

an extraction lens located just beyond the ionization chamber exit slit and an electrode opposite the exit slit (a "repeller") to extract ions from the ionization region. The extracted ions are then collimated and focused by other lenses following the extraction lens; however, the potentials applied to different lenses in the extraction system can also affect relative ion abundances [15, 29]; the extent of these effects is complex and dependent on the interaction between lenses. For reference, a schematic representation of the cross section of each cylindrically symmetrical ion extraction system is presented in Figure 3.

In one experiment, the mass spectrum of endosulfan was acquired on the HP 5985B under a "normal" set of operating potentials. The "normal" set of potentials were those optimized during instrument tuning with PFTBA/PFBN. We then analyzed the same standard under a different set of operating potentials by simply switching the potentials on the extraction and focus lenses. We found that a different mass spectrum resulted. The abundance of $\mathrm{Cl}^{-}$doubled, whereas $\mathrm{M}^{-}$ decreased by a similar proportion. This suggested that there is a disparity in transmission efficiency brought about by the lenses in the ion extraction system of the $\mathrm{HP}$ instrument, and this required further study.

A more controlled experiment was then designed to study the effects of the different tunable lenses on the transmission of low- and high-mass ions. Lowmass $\left(m / z-39\right.$, attributed to $\left.\mathrm{HF}_{2}^{-}\right)$and high-mass $(m / z-452$ and -633$)$ ions were selected from the PFTBA/PFBN tuning mixture. The transmission of another low-mass ion, $m / z-17$ (attributed to $\mathrm{OH}^{-}$), was also measured in this study. The abundances of these ions were monitored over the entire range of potentials available on each lens. This experiment was carried out on both instruments, and transmission profiles for each selected ion were then plotted as a function of lens potential.
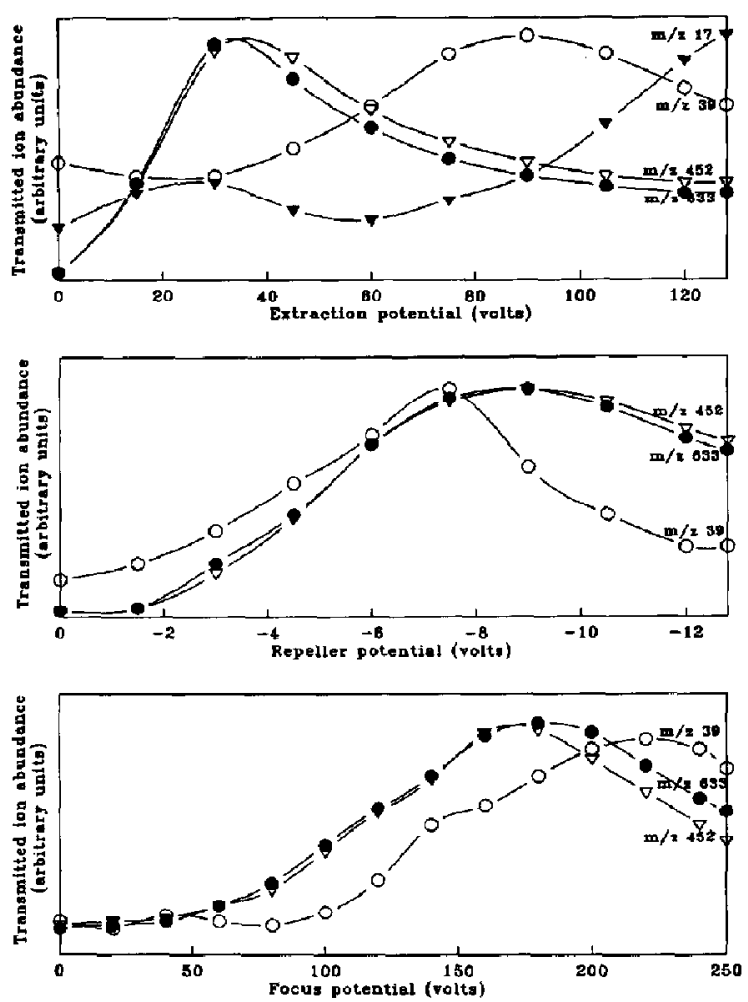

Figure 4. Detected ion abundance as a function of extraction lens (top), repeller (middle), and focus lens (bottom) potentials, all on the HP 5985B instrument.

On the HP 5985B, the potential on the extraction lens significantly affected relative abundances; Figure 4 (top) shows the transmission profiles of the ions studied as a function of the extraction lens potential. At $30 \mathrm{~V}$, the high-mass ions were effectively transmitted, but there was a distinct suppression of $m / z-39$ and $-17\left(\mathrm{OH}^{-}\right)$. Similar examination of dependence on the repeller voltage (Figure 4 , middle) did not reveal any significant differences in transmission efficiency when the potential on this electrode was varied down to approximately $-7 \mathrm{~V}$. Differences in transmission efficiency only appeared at more negative repeller potentials, which caused a significant reduction in the transmission of the low-mass ion $(m / z-39)$. There was a slight difference in the transmission efficiencies of low- and high-mass ions as a function of the focus lens potential, as indicated in Figure 4 (bottom); however, the effect was not as severe as that exhibited by the extraction lens if an operating potential of approximately $200 \mathrm{~V}$ was applied to the focus lens. Two other tunable parameters, the entrance and $X$-ray lenses (the $\mathrm{X}$-ray lens is located beyond the mass analyzer and is not shown in Figure 3) did not induce any variations in ion transmission when varied around typical operating values of $110-130 \mathrm{mV} / \mathrm{Da}$ and $5 \mathrm{~V}$, respectively.

The same experiments were carried out on the tunable lenses involved in the extraction of ions into the 
mass analyzer of the VG 30-250. The same tuning mixture of PFTBA/PFBN was used, and the low- and high-mass ions were the same. The transmission profiles for the ions, regardless of their mass, exhibited maxima at matching potentials for each lens; no variations in ion transmission efficiencies were observed on this instrument. Typical operating potentials were $0 \mathrm{~V}$ (repeller), $50 \mathrm{~V}$ (extraction), $28 \mathrm{~V}$ (focus), and $5 \mathrm{~V}$ (entrance).

It was interesting to note that the HP instrument entrance lens scans upward with mass. This lens scanning feature was disabled to study its effects on mass spectral appearance. A power supply was used instead to provide a fixed potential on the entrance lens while operating the mass spectrometer. Mass spectra were acquired at potentials of 5,50, and $100 \mathrm{~V}$ and compared with those obtained with the lens scanning normally. We found that higher potentials favored the transmission of high-mass ions. For example, the abundance of endosulfan $\mathrm{Cl}^{-}$ions did not increase significantly on going from 5 to $100 \mathrm{~V}$, but the abundance of $\mathrm{M}^{-}$ions doubled. This result suggested that the scanning feature tends to enhance the transmission of high-mass ions when used normally. Simply disabling the entrance lens scanning did not cause the HP and VG instrument spectra to become similar; however, this lens scarning feature, when used in conjunction with the other lenses in the ion extraction system of the HP instrument, may contribute to mass spectral irreproducibility among different instruments.

SIMION, an ion trajectory computation program [30], was used to examine the transmission efficiencies of low- and high-mass ions in the HP and VG instruments. Trajectories were computed for ions exiting the ion source at different points along the diameter of the exit orifice. Two specific masses, $m / z-39$ and -452 , were used to simulate low- and high-mass ions. To assign starting kinetic energies to the ions, we assumed that all ions and neutral gas molecules exit the ionization chamber with the flow of the reagent gas [31]. We further assumed that all ions start out with a kinetic energy of $0.05 \mathrm{eV}$, corresponding to a thermal energy of $100^{\circ} \mathrm{C}$ (the ion source temperature). The potentials assigned to the HP instrument lenses, for $m / z-39$ ions, were $-7 \mathrm{~V}$ (repeller), $30 \mathrm{~V}$ (extraction), $200 \mathrm{~V}$ (focus), and $5 \mathrm{~V}$ (entrance). For $m / z-452$ ions, the potentials were $-7,30,200$, and $100 \mathrm{~V}$, respectively. These values were used because results from the entrance lens experiment, discussed in the preceding section, suggested that the detected abundances of low- and high-mass ions under lens scanning conditions were similar to those obtained at fixed entrance lens potentials of 5 and $100 \mathrm{~V}$, respectively.

Figure 5 (top left and right) shows the trajectories of low- and high-mass ions through the HP 5985B system. A striking feature is observed when comparing the trajectories of low- and high-mass ions under typical operating potentials; the shapes at the end point (the point where the ion beam enters the mass analyzer) are very different. The resulting trajectories indi- cate that low-mass ions are more defocused (a more divergent beam) as they travel through the entrance lens into the mass analyzer (Figure 5, top left). Although the ion acceptance characteristics of the mass analyzer are not well known, a slightly converging and focused beam of ions is preferred for best resolution and sensitivity [32]. Our SIMION calculations suggest that low-mass ions are less well collimated, and therefore their insertion into the quadrupole field may be inherently unfavorable under normal conditions on this instrument. This may account for the low signals seen for low-mass ions.

A similar SIMION investigation of the transmission of low- and high-mass ions was also carried out for the VG 30-250 ion extraction system. Low-mass $(m / z-39)$ and high-mass $(m / z-452)$ ions were again started with initial kinetic energies of 0.05 . The potentials assigned to the lenses were $0 \mathrm{~V}$ (repeller), $50 \mathrm{~V}$ (extraction), $28 \mathrm{~V}$ (focus), and $5 \mathrm{~V}$ (energy). Figure 5 (bottom left and right) shows the trajectories of low- and highmass ions as they travel through the VG instrument system. Note that there were negligible differences in the ion beam trajectories of high-and low-mass ions as they enter the prefilters. Because both low- and highmass ions enter the analyzer with the same degree of collimation, they should be transmitted to the mass analyzer with similar efficiencies.

\section{Conclusions}

Small differences in ion source temperature and pressure may not always be responsible for ECNI mass spectral irreproducibility among different irstruments. A comparison of detection limits of endosulfan when only high-mass ions were used for quantitation indicated that similar amounts of high-mass ions were generated in the ion source of the HP $5985 B$ and VG 30-250 instruments. In this particular case, variations in the ion transmission efficiencies of the extrac-

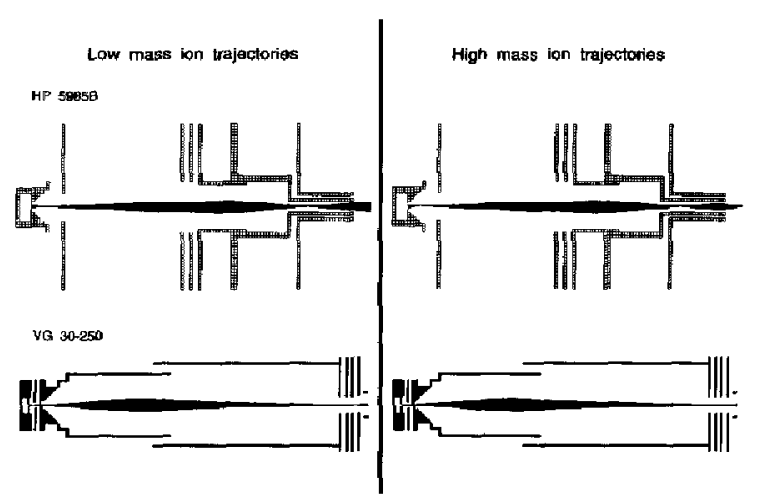

Figure 5. Computed trajectories for low-mass ions (left) and high-mass ions (right) through the HP $5985 \mathrm{~B}$ (top) and $V G$ 30-250 (bottom) instrument ion extraction systems. In this case, the low-mass ions were $m / z-39$, and the high-mass ions were $m / z-452$. 
tion optics caused the mass spectral "irreproducibility" of relative ion abundances. Although the results of this investigation indicate that extraction of low-mass ions in the HP instrument is suppressed under typical operating potentials, it by no means represents a weakness in the instrument. In fact, we have used it successfully for ECNI work in the past. Because little useful information can be obtained from the presence of a high abundance of low-mass ions, the HP instrument system is inadvertently optimized to enhance the transmission of high-mass ions for maximum sensitivity.

\section{Acknowledgments}

We thauk Mark Dearth (now of the Ford Mutor Cu.) for important preliminary experiments and calculations; Eric Grimsrud (Montana State University), Kelsey Cook (University of Tennessee), and Tom Merren (VG Biotech) for helpful discussions; Paul Goodley (Hewlett-Packard Corp.) for providing the alternate magnets for the HP 5985B and for other helpful suggestions; and Jeff Wallace for technical assistance. This work was supported by the U.S. Department of Energy through grant 87ER-60530.

\section{References}

1. Stemmler, E. A.; Hites, R. A. Anal. Chem. 1988, 60, 787-792.

2. Stemmler, E. A.; Ilites, R. A. Biomed. Mass. Spectrom, 1987, 14, 417-434.

3. Dougherty, R. C. Anal. Chem. 1981, 53,625A-636A.

4. Dougherty, R. C.; Roberts, T. D.; Biros, F. I. Anal. Chem. 1975, 47, 54-59.

5. Hunt, D. F; Crow, F. W. Anal. Chem. 1978, 50, 1781-1784.

6. Kuehl, D.; Whitaker, M. J.; Dougherty, R. C. Anal. Chem. 1980, 935-940.

7. Moyer, J. R.; Elder, J. L. J. Agric. Food Chem. 1984, 32, 866-868.

8. Ramdahl, T; U/rdal, K. Anal Chem. 1982, 54, 2256-2260

9. Hermanson, M. H.; Hites, R. A. Environ. Sci. Technol. 1990, $24,666-671$.

10. Eitzer, B. D.; Hites, R. A. Environ. Sci. Technol. 1989, 23, $1389-1395$.

11. Swackhamer, D. L.; Hites, R. A. Environ. Sci. Technol. 1988, $22,543-548$.

12. Oehme, M.; Stockl, D.; Knoppel, H. Anal. Chem. 1986, 58, 554-558.
13. Stockl, D.; Budzikiewicz, H. Org. Mass. Spectrom. 1982, 17, $470-474$.

14. Stemmler, E. A.; Hites, R. A.; Arbogast, B.; Budde, W. L.; Deinzer, M. L.; Dougherty, R. C.; Eichelberger, J. W.; Foltz, R. L.; Grimm, C.; Grimsrud, E. P.; Sakashita, C.; Sears, L. I. Anal. Chem. 1988, 60, 781-787.

15. Stemmler, E. A. Ph.D. thesis, Indiana University, 1986.

16. Siegel, M. W. Int. J. Mass. Spectrom. Ion Phys. 1983, 46, 325-328.

17. Kruger, W.; Kuypers, N.; Michnowicz, J. Proceedings of the 21st Annual Conference on Mass Spectrometry and Allied Topics; San Francisco, CA, May 20-25, 1973; pp. 381-384.

18. Christophorou, L. G.; Macorkle, D. L.; Christodoulides, A. A. Electron Molecule Interactions and Their Applications, Vol. 1; Christophorou, L. G., Ed.; Academic: New York, 1984, pp. 477-617.

19. Hunt, D. F.; Crow, F. W. Proceedings of the 9th Materials Research Symposium, April 1978, NBS Special Publication 519, National Bureau of Standards, Washington, DC, 1979, pp. 601-607.

20. Christophorou, L. G,; Compton, R. N.; Hurst, G. S.; Reinhardt, P. W. J. Chem. Phys. 1966, 45, 536-547.

21. Naff, W. T.; Compton, R. N.; Cooper, C. D. J. Chem. Phys. 1971, 54, 212-222.

22. Stemmler, E. A.; Hites, R. A. Biomed. Environ. Mass. Spectrorn. 1988, 15, 659-667.

23. Greaves, J.; Bekesi, J. G.; Roboz, J. Biomed. Environ. Mass Spectrom. 1982, 9, 406-410.

24. Garland, W. A.; Miwa, B. J. Biomed. Environ. Mass Spectrom. 1983, 10, 126-129.

25. Laramee, J.; Deinzer, M. Proceedings of the 33rd ASMC Conference on Mass Spectrometry and Allied Topics, San Diego, CA, May 26-31, 1985; pp. 87-88.

26. Gregor, I. K.; Guilhaus, M. Int. I. Mass. Spectrom. Ion Processes, 1984, 56, 167-176.

27. Szulejko, J. E.; Howe, I.; Beynon, J. H.; Schlunegger, U. P. Org. Mass Spectrom. 1980. 15, 263-267.

28. Goodley, P. Hewlett-Packard, Scientific Instrument Division, Palo Alto, CA 94304, personal communication,

29. Rullkotter, J-; Budzikiewicz, H. Int. I. Mass Spectrom. Ion Phys. 1976, 20, 269.

30. Dahl, D. A.; Delmore, J. E. SIMION Version 4.1, Idaho National Engineering Laboratory, EG \& G Idaho Inc. MS 2208, P.O. Box 1625, Idaho Falls, ID 83415.

31. Castleman, Jr., A. W.; Keesee, R. G. Chem. Rev. 1986, 86, 589-618.

32. Dawson, P. H.; Bingqi, Y. Int. J. Mass Spectrom. Ion Processes $1983,54,159-168$. 